Bibliotecas. Vol 38, №2, julio - diciembre, 2020. EISSN: 1659-3286

URL: http://www.revistas.una.ac.cr/index.php/bibliotecas/index

DOI: http://dx.doi.org/10.15359/rb.38-2.1

Licencia: Creative Commons (BY-NC-SA) 4.0 Internacional

\title{
Design Thinking para la docencia universitaria en bibliotecología
}

\author{
Design Thinking for the Teaching of Library and Information Sciences
}

\author{
Daniela Rodríguez Valerio \\ Universidad de Costa Rica
}

ORCID ID: https://orcid.org/0000-0003-3737-8851

Recibido: 29 de setiembre de $2019 \quad$ Aceptado: 13 de marzo de 2020

Publicado: 03 de julio de 2020

\begin{abstract}
Resumen:
Este estudio tiene como objetivo presentar la aplicación de la metodología design thinking o pensamiento de diseño en escenarios de enseñanza e investigar sobre estrategias implementadas en el marco de la docencia universitaria en bibliotecología. El trabajo se ha realizado mediante la revisión de artículos, libros, sitios web de organizaciones que lideran en el tema y actas de conferencias encontradas en bases de datos académicas. Se halló que existen numerosas evidencias de su implementación en docencia y con resultados satisfactorios; sin embargo, hay poca información sobre iniciativas en la enseñanza de la bibliotecología y en idioma español, a pesar de que es una metodología que se puede implementar en la enseñanza de la disciplina para fomentar estrategias didácticas constructivistas.
\end{abstract}

Palabras clave:

Design thinking, pensamiento de diseño, bibliotecología, enseñanza universitaria, estrategia constructivista

\begin{abstract}
:
The study presents the application of the design thinking methodology in teaching scenarios and research on strategies implemented in the framework of university teaching in librarianship. The work has been done through the review of articles, books, websites of leading organizations in the field and conference proceedings found in academic databases. It was found that there is a lot of evidence of its implementation in teaching and with satisfactory results; however, there is little information about initiatives in the teaching of librarianship and in Spanish language in spite of the fact that it is a methodology that can be implemented in the teaching of the discipline to promote constructivist didactic strategies.
\end{abstract}

\section{Keywords:}

Design thinking, library science, university teaching, constructivist strategy 


\section{Introducción}

Desde hace algunos años se ha venido apostando por modelos educativos menos conductuales que favorezcan la participación activa del estudiante y que estimulen la creatividad. En la docencia universitaria, particularmente en el caso de la enseñanza de las Ciencias Sociales, es común disponer de escenarios que propician el desarrollo de este tipo de modelos más dinámicos, es por esto que se propone el uso de la metodología design thinking o pensamiento de diseño como estrategia para construir el conocimiento y promover la participación e interacción de los estudiantes, específicamente en la enseñanza de la bibliotecología.

El design thinking comprende una metodología que, en cinco etapas, permite conocer los problemas, definirlos, idear soluciones, hacer prototipos de las ideas para conocer si son factibles o refinarlas y finalmente probarlas. Es una metodología que nació en las industrias creativa y empresarial para el desarrollo de productos y estrategias innovadoras; sin embargo, con el pasar de los años se ha venido adoptando en otros espacios como el diseño de servicios y en el campo educativo a modo de una ruta de aprendizaje.

En educación, esta metodología ha despertado interés, pues promueve la adopción de conocimientos, el aprendizaje significativo y el desarrollo de competencias clave para desenvolverse en el contexto profesional actual, tales como creatividad, comunicación, aprender a aprender, trabajo colaborativo y solución de problemas.

En este artículo, se exponen estrategias didácticas desarrolladas en la docencia universitaria que tienen como eje principal design thinking. Asimismo, se presentan algunos casos aplicados específicamente en la bibliotecología, una disciplina que se caracteriza por tener un alto componente participativo en sus procesos de enseñanza, que implica la creación de servicios, productos de información y el estudio de poblaciones usuarias, áreas en las cuales el design thinking podría aportar muchas experiencias positivas y participativas. 


\section{Design thinking}

Según la Interaction Design Foundation (2018a y 2018b) los primeros indicios de esta metodología se remontan a las décadas de 1950 y 1960 en las industrias de la ingeniería y la arquitectura, especialmente durante los inicios de 1960 cuando nace el interés por introducir bases científicas en los procesos de diseño y orientarlos a la solución de problemas complejos. Es a mediados de los años 1960 que el teórico Horst Rittel introdujo el término wicked problems o problemas complejos a través de un estudio enmarcado en el área del diseño dirigido a practicantes y académicos de este tiempo, y es este término el que motiva la creación de una metodología colaborativa para resolverlos. Más adelante, en 1969, el científico y premio nobel Herbert A. Simon es el primero en definir el diseño como una ciencia y forma de pensar. En su libro Sciences of the Artificial, se le atribuyen los principios del design thinking, ya que introdujo la importancia de la observación, el prototipado y las pruebas para crear buenos diseños; sin embargo, lo hace en un estudio sobre inteligencia artificial.

Como parte de los inicios de esta disciplina, también se destaca el aporte de Robert H. McKim quien en 1973 introduce elementos de la metodología en su libro Experiences in visual thinking, en esta obra el autor se enfoca en la idea de resolver problemas de forma holística y mediante el pensamiento visual. Más adelante, en 1987, Peter Rowe, director de los programas de diseño urbano en Harvard, publicó el libro Design thinking en el cual enfatiza la importancia del pensamiento cognitivo para la solución de problemas en el campo del diseño arquitectónico.

Sin embargo, es en 1990 cuando se da un apogeo del design thinking, gracias a los aportes de IDEO en la Escuela de Diseño de la Universidad de Stanford. IDEO es una compañía de diseño, la primera en desarrollar terminología, pasos y manuales sobre design thinking. Desde el año 2005 esta metodología se enseña en dicha universidad en lo que hoy se conoce como el Hasso Plattner Institute of Design. Esta realidad ha impactado a otras prestigiosas universidades, las cuales han adoptado la metodología y la han aplicado en otros campos más amplios, considerando que el diseño es un proceso que participa en todas las dimensiones de la vida del ser humano. 
Buchanan (1992) establece que existen cuatro grandes áreas que implican procesos de diseño y que no son desempeñadas precisamente por diseñadores, además aclara que todas estas tareas se basan en encontrar un problema y crear una solución creativa. Es por esta razón que este artículo sostiene que el design thinking se puede aplicar en procesos de enseñanza y aprendizaje. Las cuatro áreas establecidas por Buchanan (1992) son:

- Diseño de comunicación simbólica y visual (implica comunicar información)

- Diseño de objetos materiales

- Diseño de actividades y servicios con el fin de convertirlas en experiencias agradables

- Diseño de espacios destinados al juego, trabajo, aprendizaje y diario vivir

Luego de haber establecido una breve reseña sobre los orígenes de design thinking, es necesario precisar en qué consiste. El design thinking es una metodología que busca solucionar problemas mediante la experimentación y la generación de ideas innovadoras, también posibilita el diseño o rediseño de productos o servicios, tal como lo presentan Stickdorn y Schneider (2011). Estas ideas y soluciones se consiguen a través de cinco etapas: empatizar, definir, idear, prototipar y evaluar; a continuación, se define cada una de ellas de acuerdo con Hasso Plattner Institute of Design at Stanford (s.f.) y a Stickdorn y Schneider (2011):

- Empatizar: los diseños, servicios y productos deben crearse basados en las necesidades de la población usuaria, por lo tanto, se le debe observar, escuchar y comprender. Esta etapa de empatía va a permitir conocer al usuario a profundidad para generar auténticas soluciones más adelante, durante la etapa "idear". La entrevista a profundidad, la observación, el customer journey, storyboards, expectation maps, role plays, personas, preguntar qué, cómo y por qué, además los grupos focales son técnicas adecuadas para esta etapa. A continuación, se explican algunas de estas estrategias:

Customer journey: esta estrategia permite explorar procesos, por lo cual es orientada al diseño de servicios. Consiste en bosquejar visualmente los diferentes pasos que una persona usuaria debe realizar para aprovechar un servicio. Esta estrategia se puede realizar con los propios usuarios o con las 
personas encargadas de brindar el servicio que se está analizando. Al plasmar los pasos de forma visual es más sencillo enterarse de problemas durante el proceso o detectar puntos de mejora.

Storyboards: consiste en proponer una situación real en la que una persona haría uso de un servicio, para posteriormente representarla con ayuda de dibujos o fotografías. Lo más usual es que esta representación se muestre detalladamente en forma de tira cómica para ayudar a contar una historia. El propósito de esta estrategia es estudiar cómo es la experiencia de un usuario al interactuar con el servicio. El uso de dibujos posibilita que quienes están diseñando un servicio se pongan en los zapatos de las personas usuarias y se inicie un espacio de discusión.

Expectation maps: son herramientas de diagnóstico que se ejecutan con la comunidad usuaria real y potencial de un servicio. Se pueden realizar por medio de talleres en los cuales las personas plasman lluvias de ideas de forma gráfica y expresan cómo sería el servicio ideal. Esta estrategia permitirá descubrir las expectativas de los usuarios y comprender qué debe hacer una organización para realmente satisfacer sus necesidades.

Role play: esta es una estrategia que se lleva a cabo con las personas encargadas de brindar el servicio, para que analicen su trato y desenvolvimiento con las personas a quienes deben brindar un servicio. Se les motiva a recrear de forma "teatral" situaciones comunes que pueden vivir con un usuario al realizar un determinado proceso. Las personas participantes pueden intercambiar los roles entre sí y debe haber otras observando para discutir y brindar retroalimentación. Esta estrategia se puede acompañar con cartas donde se anoten los roles y situaciones de forma detallada, en las que se especifican estados de ánimo, características personales y problemas.

Personas: esta es una estrategia que facilita estudiar al usuario, comprenderlo y representarlo. Consiste en crear perfiles ficticios de personas usuarias de un servicio o personajes que representan los intereses de un determinado grupo. Por lo tanto, se debe investigar al usuario a profundidad. A pesar de que el personaje o perfil es ficticio, el grupo que representa y su realidad sí es real. 
Presentar estos perfiles a las personas desarrolladoras de un servicio o producto es de utilidad para iniciar discusiones grupales, identificar oportunidades e innovar.

- Definir: en esta etapa se determinan el problema y el desafío del proyecto, basándose en lo aprendido de la persona usuaria y su contexto. Esto será posible gracias a la observación, análisis y síntesis de la información recolectada durante la etapa de empatía, de allí que esta primera etapa busque ser muy detallada. El desafío debe ser motivante, significativo y claro para el equipo. Se debe considerar además que sea discutible y evaluable.

- Idear: posterior a la definición de un problema o desafío se inicia un proceso de generación de la máxima cantidad de ideas para solucionarlo o tratarlo. Todas las ideas son válidas y se consideran aquellas que son racionales y también las que no lo son, ya que en esta etapa lo que se persigue es innovar y pensar en soluciones creativas a los problemas. En este proceso se trabaja con brainstorms o lluvias de ideas guiadas por un moderador y se debe procurar plantear varias soluciones, esto estimula el trabajo colectivo, descubrir áreas inesperadas de exploración y analizar la visión de los equipos de trabajo.

- Prototipar: una vez que se tienen soluciones al desafío o problema, los equipos deben trabajar en construir un prototipo o varios de la idea que mejor resuelve la situación. Un prototipo puede ser un dibujo o un artefacto con el que se puede interactuar para acercarse de una forma bastante real a la solución. Por lo tanto, en esta etapa a los equipos idealmente se les debe dotar de herramientas, materiales, objetos, recursos tecnológicos y de un espacio adecuado para que puedan dibujar, diseñar y/o crear prototipos de manera rápida. Sin embargo, para hacer design thinking puede ser suficiente facilitar papel, cartón y materiales reciclados, ya que lo que busca es que el equipo pueda crear, experimentar, colaborar y explotar su creatividad. El prototipo debe permitir comunicar la idea, facilitar cometer errores y evaluar ideas mientras se construye. Puede ir más allá de un objeto ya que puede tratarse de un sitio web, un sistema, un espacio, un negocio, un servicio, entre otros. 
- Evaluar: para llegar a esta etapa es indispensable contar con el prototipo diseñado en la etapa anterior, el cual será expuesto a otras personas en contexto y a quienes estaría dirigido para que puedan interactuar con él, experimentar y solicitar su retroalimentación. Esta es otra oportunidad para retomar la primera etapa y hacer "empatía"; sin embargo, el objetivo terminal de este proceso es evaluar las soluciones para refinarlas y mejorarlas. Evaluar permite conocer más sobre la persona usuaria, crear experiencias, revelar necesidades potenciales, mejorar y crear soluciones realmente dirigidas a la población usuaria.

Como parte de la metodología design thinking o pensamiento de diseño, es importante mencionar el concepto de "iterar", que significa "repetir". Esto quiere decir que la metodología incentiva a comenzar el proceso de nuevo o repetir una de sus etapas para corregir un prototipo ya evaluado o para mejorarlo.

\section{El Modelo pedagógico constructivista y el design thinking}

En un contexto educativo cada vez más abierto, que promueve la participación activa del estudiantado y con una realidad laboral que exige profesionales que "aprendan a aprender", los centros educativos han apostado por adoptar modelos pedagógicos menos conductuales y que sitúen al estudiante como un individuo activo y constructor de su propio conocimiento. Por lo tanto, es importante mencionar la relevancia del modelo pedagógico constructivista, expuesto principalmente por "Jean Piaget (1896-1980), Lev S. Vigotsky (1896-1934), Lawrence Kohlberg (1927-1987), George Kelly (1905-1967), David Ausubel (1918-2008) y Joseph Novak (1932-)" (Vergara y Cuentas, 2015, p. 927).

La intencionalidad de este modelo es alcanzar una comprensión cognitiva en la que el individuo aprenda mediante la interacción con el ambiente y sí mismo, por lo cual se favorece la actividad, el diálogo, los talleres, los laboratorios, la exploración, investigación, reflexión, los estudios de casos y el debate. También, como parte del proceso de construcción de su conocimiento, se fomentan la duda, el error y la interacción social, además se propicia el aprendizaje significativo, el cual proviene de la interacción entorno-vivencia-interés-reflexión. 
En cuanto a los contenidos, se privilegian especialmente la formación de conceptos, la interpretación de datos y la aplicación de principios (Vergara y Cuentas, 2015). También, es muy destacable la importancia de la interacción con otros individuos para un aprendizaje óptimo, ya que: "la forma de aprender de los humanos es altamente social y es mediante el aprendizaje colaborativo o cooperativo como se optimizan los procesos de memoria, atención y análisis" (Mora, 2010, p. 105).

Por lo tanto, conociendo qué es el design thinking y el modelo pedagógico constructivista, es posible detectar puntos de convergencia entre ambos; por ejemplo, la importancia de la interacción social entre individuos para construir conocimiento y compartir ideas, el papel activo del individuo al tener iniciativa y soluciones para los problemas, los procesos de construcción y el rol del error para promover el conocimiento y la solución creativa de problemas. Al respecto, Koh, Chai, Wong y Hong (2015) mencionan que el diseño es la principal actividad pedagógica del constructivismo y a través de este los estudiantes se comprometen más con el aprendizaje, pues hablan, piensan, imaginan y construyen su conocimiento. Los autores, además, citan a Scardamalia (2002) quien estableció ciertos principios del diseño que se adecúan al enfoque de construcción de conocimiento, algunos de ellos son: enfrentarse a problemas reales de interés para los estudiantes, articular teorías e ideas y mejorarlas de forma colectiva mediante el discurso.

Cabe recalcar que la metodología design thinking requiere que los participantes conozcan investigar y explorar, lo cual conlleva a la generación de competencias que se enmarcan en el modelo pedagógico constructivista, se introducen en espacios de debate y construcción en donde deben tener iniciativa y creatividad, respetar ideas y convivir con otros individuos, lo cual estimula competencias relacionadas con su desarrollo social y personal.

Otro punto en el que convergen esta metodología y el modelo pedagógico constructivista recae en el aprendizaje basado en problemas (ABP). Al respecto, Lor (2017) establece que el ABP parte de un problema propuesto por el profesor, que debe explorarse y solucionarse utilizando los conocimientos adquiridos en el aula, lo cual responde a algún objetivo de aprendizaje proveniente del plan de estudios. Por lo tanto, el design thinking es una 
metodología que se adapta y que incluso ofrece mejores posibilidades, ya que en este el individuo es quien define el problema basándose en su propia exploración. El autor explica que en los últimos años se ha estado estudiando esta metodología en el marco de la educación, y resalta que ya existen estudios que abordan su utilización para el diseño de currículum, como estrategia de enseñanza y aprendizaje y para la formación de profesores.

\section{Design thinking y docencia universitaria}

Dado que la docencia universitaria implica procesos de innovación, creatividad y solución de problemas, el design thinking constituye una metodología que puede aplicarse tanto en el aula como en otras actividades propias del quehacer docente. A través de una amplia revisión de literatura Lor (2017) encontró usos en diseño curricular y formación de profesores. Gardner (2017a) expone un caso donde Madlen Simon, Decana de Asuntos Académicos de la Escuela de Arquitectura, Planificación y Preservación de la Universidad de Maryland, recurrió a esta metodología para llegar a acuerdos sobre una política para manejar las quejas de los estudiantes contra la Facultad; la política al principio generó mucha negatividad entre el profesorado, sin embargo, a través de procesos de empatía, se lograron determinar las preocupaciones de los implicados y llegar a acuerdos para finalmente aprobarla. También cuenta sobre un grupo de 50 profesores que se reunieron en la Universidad de Stanford para aprender sobre design thinking a través de un Teaching and learning studio, de este taller se generó la idea de crear una solución de aprendizaje para estudiantes de avanzada edad. Gardner (2017a) detalla que las ideas innovadoras suelen resultar descabelladas para muchos académicos, pero cuando se exponen y conversan debidamente es posible romper esquemas y demostrar su viabilidad, es así como surgen las innovaciones.

En los procesos de enseñanza y aprendizaje, Gardner (2017b) establece que el design thinking es factible dependiendo del curso y de los objetivos, si el curso requiere de respuestas correctas y soluciones óptimas, esta metodología no es la adecuada, pero cuando hay oportunidad de organizar sesiones de lluvia de ideas, será una estrategia idónea, considerando que la metodología ofrece el tipo de aprendizaje que los estudiantes prefieren, según evidencias. Menciona Gardner (2017b) que se puede utilizar cuando el estudiantado 
tiene dificultades para trabajar en equipo o terminar el trabajo en clases, dado que las actividades se realizan con tiempos establecidos por el moderador. Por otra parte, desarrollar estrategias didácticas con design thinking implica estar en un ambiente donde las personas saben que pueden experimentar, tomar riesgos y explorar (Leverenz, 2014).

Más allá de la enseñanza del diseño, también hay evidencias sobre su uso en la enseñanza de la medicina, Newberry (2016) explica sobre su aplicación en un curso introductorio con estudiantes millenials que usaron design thinking para involucrarse con la comunidad, realizar trabajo de campo y plantear estrategias de prevención de enfermedades. La estrategia logró un alto compromiso de los estudiantes, quienes mantuvieran una alta asistencia a las clases y durante la evaluación del curso muchos mencionaron la importancia de contar con evaluaciones más estructuradas que los conectaran más con su rol como futuros médicos.

También, en la Universidad de Amsterdam, se detectó que los estudiantes tenían dificultades para desarrollar el pensamiento crítico, la solución creativa de problemas relacionados con el paciente y la comunicación efectiva. Entonces se organizó un curso con 27 estudiantes de medicina, psicología, neurociencias, ciencias sociales y arte, a quienes se les dividió en grupos y se les asignó un caso de estudio que debían resolver de forma creativa, colaborativa y con pacientes reales. Entonces, mediante el design thinking crearon soluciones innovadoras y consideradas relevantes por los pacientes. Sobre la evaluación del curso, el estudiantado apreció el desarrollo académico obtenido y la adquisición de destrezas para el trabajo colaborativo y creativo; consideraron negativa la falta de supervisión y las dificultades para trabajar con profesionales de otras disciplinas (Van de Grift y Kroeze, 2016).

Además, se tienen evidencias sobre el uso en la enseñanza de la escritura académica. Leverenz (2014) establece que escribir es un acto de crear, que se requiere creatividad y por esto utilizó la metodología para enseñar a sus estudiantes. El autor explica que en un curso asignó a sus estudiantes investigar un problema en equipos y diseñar una solución. Seguidamente, les mostró un video en que un equipo de IDEO diseñaba un carrito para compras, en el cual se mostraban las etapas del design thinking y les explicó que ellos harían lo mismo. Por lo tanto, iniciaron investigando sobre su problema para definirlo y familiarizarse 
con él, posteriormente identificaron una audiencia potencial para su diseño, después se organizó una sesión de lluvia de ideas para proponer soluciones y se crearon tres prototipos de su diseño, los cuales fueron sometidos a votación por el resto de la clase, eligiendo el que se ajustaba mejor a la solución del problema, creando un borrador que se pudiera probar con usuarios potenciales, para revisarlo y presentarlo nuevamente, esta vez ante tres jueces imparciales que votaron por el diseño más convincente. A pesar de que el producto de esta actividad no fue escrito, su proceso de diseño fue documentado posteriormente en un artículo, incluyendo un contrato, un plan de investigación y bibliografía, incluso los estudiantes tuvieron que escribir un borrador de su proyecto. Con esta metodología, los participantes aprendieron a investigar sobre su problema, diseñar una solución, trabajar en equipo y desarrollaron habilidades de escritura y comunicación que les acercaron a la realidad de un investigador que escribe por un propósito: divulgar la ciencia.

Hay iniciativas del uso de design thinking para la enseñanza de negocios en estudiantes de pregrado, específicamente para incentivar la competencia de innovación que deben tener estos profesionales. Armstrong (2016) comenta que para implementar esta metodología se motivó a los estudiantes a rediseñar su experiencia en la universidad. Iniciando con la etapa de empatía cada estudiante entrevistó a un compañero sobre cómo había llegado a la universidad, sus motivaciones y expectativas. Esto lo debían anotar y después tuvieron oportunidad para tener una segunda entrevista y clarificar información. Seguidamente los estudiantes tuvieron que determinar cuáles eran los objetivos y motivaciones principales de su compañero y con base en ello encontrar y anotar un problema que solucionar. Después, conscientes del problema, escribieron muchas soluciones sin importar si eran viables o no, para presentarlas a otro compañero y obtener retroalimentación. Una vez con esta información, procedieron a repensar una solución y a crear un prototipo de esta con el que su compañero pudiera interactuar y, de esta manera, evaluar el prototipo para tener más retroalimentación y plantear una solución final acorde con las necesidades de su compañero.

El campo de la pedagogía también refleja interés en la metodología design thinking. Luka (2014) establece que el conocimiento es asimilado a través de la acción y mediante procesos analíticos y sintéticos, en el cual el ciclo de aprendizaje ideal comprende las siguientes fases: 
experimentar, reflexionar, pensar y actuar, todo ello comprendido por el design thinking a través de sus cinco etapas (Beckman y Barry, 2007). El autor detalla que en una fase analítica de aprendizaje el estudiante parte de sus conocimientos, observa y experimenta para plantear soluciones basadas en los preceptos teóricos, para posteriormente pasar a la fase sintética y poner en práctica sus conocimientos, lo cual se ve reflejado en el design thinking como estrategia didáctica.

En el nivel latinoamericano no se encontró mucha literatura científica que aborde el design thinking en la educación, sin embargo, es relevante mencionar que en Brasil existe una iniciativa que promueve su implementación en este campo, que puede consultarse por medio del enlace https://www.dtparaeducadores.org.br/site/; además, IDEO creó un manual propiamente para educadores, el cual está traducido a varios idiomas para promover su utilización (IDEO, 2013).

\section{El proceso de aprendizaje en la bibliotecología}

La bibliotecología es una ciencia social que se encarga de la organización de la información en todos sus formatos, se ocupa de garantizar su acceso y de hacerla recuperable para transformarla en conocimiento. Entonces, todos estos procesos envuelven gran cantidad de enseñanzas que van desde los fundamentos de la profesión, el procesamiento técnico de la información, hasta la atención de usuarios, el diseño y gestión de sistemas, el manejo de tecnologías, la administración de centros de información, el análisis estadístico de la información para la toma de decisiones, el desarrollo y gestión de colecciones, el mercadeo de la información, los procesos de investigación, entre muchas otras áreas que implican el trabajo con un recurso de tanta importancia como lo es la información. Además, la bibliotecología tiene un alto componente social, por lo cual en su proceso de garantizar el acceso a la información promueve las actividades de inclusión social y de fomento y animación a la lectura.

Se recalca que es una disciplina en constante evolución, proyectada a trabajar de acuerdo con las necesidades sociales del entorno. En el contexto actual, Rosa y Storey (2016) 
explican que se están experimentando retos y oportunidades relacionados con los cambios en la población, innovaciones en los modelos de enseñanza y aprendizaje, por las necesidades de alfabetización digital y de inclusión, por los cambios en la naturaleza de las colecciones, la computación en la nube, la reducción de presupuestos y recursos para las bibliotecas y la creciente globalización y socialización de la información. Por lo tanto, la bibliotecología ha tenido que incursionar en áreas como la alfabetización mediática e informacional, brindar acceso a contenidos en formatos alternos al impreso, emprender proyectos e iniciativas de cooperación entre bibliotecas, la curación de contenidos, la adopción de nuevas tecnologías como los dispositivos móviles para el procesamiento de datos (como el big data) y los datos enlazados o linked data, entre otros.

En su proceso de aprendizaje, la bibliotecología presenta una gran tradición teórica y un vasto componente práctico. Ríos-Ortega (2008) establece que, en el ejercicio de su profesión, el bibliotecólogo "sigue necesariamente una teoría acerca de la mejor manera para lograr una meta bibliotecológica deseable" (p. 8); esto puede visualizarse en los procesos de desarrollo de colecciones, en su labor de orientación a la población usuaria en la búsqueda de información y en la utilización de normas y estándares para el tratamiento de la información. Dicho autor explica que mediante la práctica estas teorías se reformulan o perfeccionan, por lo tanto, no es posible aprender bibliotecología sin ambos componentes. Es por esta razón que para el aprendizaje de la disciplina es necesario emplear estrategias didácticas que le permitan al estudiante asimilar los conocimientos, contextualizarlos en su ámbito profesional y llevarlos a la práctica.

Otra razón por la que es importante la teoría se debe a la realidad de las bibliotecas. Sabor (1968) explica que al menos en América Latina las bibliotecas muchas veces cuentan con pobreza técnica y administrativa, lo que impide utilizarlas como modelo de lo que debería ser la práctica del bibliotecólogo. Por lo tanto, es fundamental dotar a los estudiantes de los fundamentos básicos de la disciplina y de estrategias metodológicas como design thinking para que el futuro profesional tenga las herramientas y habilidades necesarias para mejorar las estructuras existentes. 
Algunas técnicas que se consideran efectivas para la enseñanza y aprendizaje de la bibliotecología son asignación de lecturas, organización de grupos de discusión, asignación de proyectos, tutorías, simposios, observación de campo, exposiciones del docente, consultas bibliográficas, catalogación de textos, discusiones en clase, trabajos prácticos o en laboratorios, elaboración de monografías y bibliografías, entrevistas, visitas y estudio de casos. Además, desde los años 1970 se ha privilegiado el uso de medios electrónicos para el aprendizaje de los bibliotecólogos, debido a la relevante relación bibliotecología-tecnología. Aunado a lo anterior, deben destacarse los cursos de metodología de la investigación, ya que estos introducen al estudiantado en el trabajo intelectual, les permite desarrollar habilidades de redacción y de trabajo autónomo, y constituir herramientas útiles para su ejercicio profesional (Pirela y Pulido, 2017; Ríos-Ortega, 2008 y Sabor, 1968).

Respecto a los planes de estudio, en Latinoamérica las mallas curriculares tienden a variar, a organizarse mediante un sistema de créditos con cursos articulados entre sí y con contenidos estipulados en programas de cursos. Sin embargo, las tendencias actuales apuntan a concebir un currículo que favorezca una formación por competencias que les permita a las personas egresadas enfrentarse de una mejor manera a la realidad laboral actual. Escobar e Infante (2018) recalcan la importancia de promover en el bibliotecólogo competencias relacionadas con la cooperación, la colaboración, el aprender a aprender, la comunicación, la capacidad de negociación, el pensamiento creativo y propositivo y la facilidad para plantear y resolver problemas.

También, la bibliotecología es una disciplina multidisciplinaria que se complementa con otras áreas, como por ejemplo: informática, administración, matemática, estadística, derecho, psicología y educación; por lo tanto, sus planes de estudio tienen gran influencia de estas áreas del conocimiento e implica que el estudiantado se relacione y trabaje de forma transdisciplinaria con personas de otras áreas. Cabe destacar que, como parte del proceso de enseñanza y aprendizaje, la universidad induce al estudiantado en otros espacios que, al fin y al cabo, contribuyen con una formación competente del egresado y que estimulan capacidades complejas, es por esto que desde las instituciones de educación superior se deben promover las dinámicas propuestas por la Organización de las Naciones Unidas para la 
Educación, la Ciencia y la Cultura (UNESCO) en el marco de la Conferencia Mundial sobre Educación Superior realizada en París durante el 2009:

- Responsabilidad social universitaria: El currículo está interrelacionado con la sociedad y su desarrollo, por lo cual el estudiante debe comprender y resolver problemas con dimensiones sociales, económicas, científicas y culturales. Para cubrir este aspecto las universidades promueven prácticas inter y transdisciplinarias, que al mismo tiempo le permiten al estudiante estar en contacto con su realidad laboral.

- Acceso, calidad y equidad: la educación superior debe ser accesible y acorde con las necesidades del contexto actual. Se exigen nuevos enfoques como la educación abierta, a distancia y el uso de tecnologías de la información y comunicación.

- Internacionalización, regionalización y mundialización: debe existir cooperación internacional en temas de educación superior que promuevan diálogo cultural, iniciativas conjuntas de investigación y de acreditación y estimulen así la calidad de la educación.

- El aprendizaje, la investigación y la innovación: las instituciones de educación superior tienen que buscar alianzas provenientes de los sectores públicos y privados para aumentar la investigación y la innovación. En este punto es muy importante resaltar que las iniciativas de innovación promueven el desarrollo local e internacional, generando bienestar para la sociedad.

\section{Design thinking en la enseñanza de la bibliotecología}

La bibliotecología es una disciplina cuyos esfuerzos siempre irán enfocados a la población usuaria, es por esta razón que en este artículo se promueve el design thinking para su enseñanza, principalmente en aquellos aprendizajes que requieren de procesos creativos y de construcción. Además, producto de la diversificación del ámbito profesional del bibliotecólogo, la sociedad hoy demanda competencias que van más allá de lo técnico, como el trabajo en equipo, la comunicación, la colaboración, la solución creativa de problemas, las habilidades de investigación, la creatividad y la empatía con la sociedad.

También, los docentes interactúan con una población estudiantil que cuenta con estilos de aprendizaje muy diversos y diferentes a los de años anteriores; muchas veces han sido 
expuestos a tecnologías desde edades tempranas, por lo cual no se puede concebir una enseñanza exclusivamente magistral, considerando que los retos cognitivos en bibliotecología implican llevar los conocimientos teóricos a la práctica.

Existen muchas evidencias del uso de design thinking para desarrollar servicios y espacios propiamente en la biblioteca, pero no hay muchas experiencias documentadas sobre su implementación en la enseñanza de la disciplina. A continuación, se presentan algunas iniciativas llevadas a cabo en Estados Unidos:

- Bowler (2014) utilizó el design thinking con estudiantes de bibliotecología escolar de la Universidad de Pittsburg con miras a promover su desenvolvimiento en makerspaces. Entonces, en el marco de una actividad llamada Bots and book challenge los estudiantes se organizaron en parejas o tríos y fueron desafiados en elegir una historia para niños y al mismo tiempo interpretarla con ayuda de un robot. Se les pidió que la historia debía poderse comprender, provocar una respuesta emocional, hacer pensar al espectador y programar al robot para que fuera expresivo. El estudiantado no sabía programar, pero la herramienta utilizada hummingbird permitía hacerlo de forma visual. Seguidamente, se les dio una explicación sobre los componentes y funcionamiento básico de la herramienta. Con estos insumos y a través de las cinco etapas del design thinking, los estudiantes conceptualizaron el proyecto y después de muchos intentos fallidos generaron sus prototipos que fueron mejorando. Esta estrategia fue satisfactoria, dado que a través de un proceso constructivo y creativo los estudiantes aprendieron a utilizar los equipos del makerspace.

- Coleman (2016) explica una actividad que desarrolló en una biblioteca escolar en Chicago, Illinois, en apoyo al currículum de un curso sobre escritura para que los estudiantes aprendieran a interpretar mejor los textos y crearan historias realmente llamativas, competencias que son necesarias para que los profesionales en bibliotecología se desenvuelvan en ámbitos educativos. Por lo tanto, utilizando el cuento "Los tres cerditos" de Paul Galdone, se inició un proceso de análisis de la historia y de comprensión de sus personajes para cumplir con la etapa de "empatía". Para la etapa de definición se analizó la versión del cuento de Susan Lowell en la cual 
los personajes son diferentes y cuya locación es en el desierto. Con este ejercicio se invitó a reflexionar sobre cómo afecta la locación a las historias y a través de una lluvia de ideas se discutió sobre cómo podrían cambiar los personajes y sus problemas si el lugar volviera a cambiar. También, analizando una versión del cuento de Corey Rosen, se analizaron y definieron las personalidades de los personajes.

Después, para la etapa "Idear", los estudiantes se enfocaron en bosquejar casas para todos los cerditos discutidos, lo cual se les permitió llenar una pizarra con notas e hicieron en grupos un dibujo de la casa ideal para los cerditos, la cual debieron explicar. En el prototipo, los integrantes usaron ciertos materiales para construir una representación de su idea, lo cual promovió la creatividad y el trabajo en equipo. Finalmente, los estudiantes expusieron sus creaciones y el proceso de diseño.

- Es importante recalcar un manual de design thinking desarrollado por las bibliotecas públicas en Aarhus (Dinamarca) y de Chicago (Estados Unidos) el cual está basado en la metodología de IDEO, pero adaptado para ser aplicado directamente por las bibliotecas con el objetivo de crear mejores servicios, herramientas y experiencias para sus usuarios (IDEO, 2014). Este manual no es propiamente para la enseñanza de la bibliotecología, pero al estar contextualizado en la disciplina se puede aplicar en los procesos de enseñanza y aprendizaje.

- Además, como se cita en Clarke y Bell (2018), desde 2015 la University of Washington Information School ofrece un curso de "Métodos de diseño para bibliotecología" dirigido a sus estudiantes de maestría donde se enseña con design thinking, también la Simmons School of Library and Information Science incorporó la metodología para enseñar investigación etnográfica de usuarios, prototipado y habilidades de diseño centradas en el usuario. La Universidad Estatal de San José y Syracuse University se encuentran también incorporando design thinking en sus programas de enseñanza de bibliotecología. 


\section{Valoraciones finales}

El design thinking es una metodología que se utiliza desde hace mucho tiempo, especialmente en las áreas del diseño y negocios, pero como el diseño es un proceso que implica la solución creativa de problemas, entonces se ha estado utilizando y adaptando en otras disciplinas y para múltiples propósitos. El campo de la educación se ha visto ampliamente beneficiado, ya que el design thinking conlleva procesos de análisis, experimentación, trabajo creativo, construcción y promoción del aprendizaje colaborativo, por lo tanto, se adecúa al desarrollo de estrategias didácticas propias del modelo pedagógico constructivista, el cual incentiva un aprendizaje significativo en los estudiantes y promueve un rol activo de su parte.

Como conlleva generar múltiples ideas para la solución de problemas, el design thinking se recalca como una oportunidad que posibilita escenarios de trabajo transdiciplinarios, generando soluciones altamente innovadoras e iniciativas de emprendimiento. Se han encontrado incluso iniciativas con un alto impacto social en cuyo proceso de desarrollo se ha utilizado el design thinking. En el caso de la bibliotecología, es importante mencionar que existen suficientes evidencias de su aplicación para el diseño de servicios y productos propiamente desde y para la biblioteca, pero es muy escasa la literatura respecto a la enseñanza de la disciplina, lo cual no sucede en otras áreas como salud, negocios y diseño. Esta situación más que una limitante se considera una oportunidad, ya que:

[...] la biblioteca tiene su fin último en el servicio que brinda a los usuarios cuando ofrece

sus productos y recursos, cuando asesora en el acceso a la información, cuando garantiza la solución de problemas frente a los cambios informativos que se producen en el interior o en el exterior de la institución, o cuando a través del trabajo interdisciplinario y colaborativo identifica intereses y responsabilidades comunes. (Monfasani, 2016, p.51)

Entonces, ya que la bibliotecología es una disciplina centrada en la persona usuaria, el design thinking se considera una metodología relevante y eficiente para su enseñanza y ejecución. Se propone además como una técnica a aplicar dentro de estudios cualitativos, específicamente en investigaciones de tipo etnográficas. 
Se recalca que como estrategia didáctica el design thinking puede promover muchas experiencias positivas; sin embargo, debe emplearse en espacios educativos donde exista confianza, respeto, apertura a ideas y donde los estudiantes estén dispuestos a participar, ya que la metodología exige un rol altamente participativo por parte del educando. En este caso, el rol del docente es de guía y mediador, que además debe establecer tiempos prudenciales que permitan cumplir con las diferentes etapas establecidas.

Para esta investigación, y desde la óptica de la enseñanza de la bibliotecología, se encontraron iniciativas de uso de design thinking en procesos de enseñanza dirigidos especialmente a bibliotecólogos escolares y en cursos de diseño, no obstante, considerando su implementación en otras disciplinas, esta metodología se podría implementar en los programas de los cursos que enseñan los siguientes contenidos:

- Arquitectura de información: como parte del desarrollo o rediseño de sitios y aplicaciones web, es ideal estudiar las necesidades de sus usuarios, sus patrones de navegación y expectativas para posteriormente analizar esta información e idear soluciones atractivas y funcionales, de las cuales pueden crearse wireframes o prototipos para ser probados antes de iniciar con el proceso de programación.

- Planeamiento de espacios de la biblioteca: esta labor del profesional en bibliotecología también requiere de conocer y escuchar a sus usuarios, visualizar problemas con la ubicación de los espacios, generar ideas creativas y usualmente elaborar maquetas físicas o con ayuda de sistemas para poder visualizar de forma realista los espacios y así someterlos a consideración.

- Mercadeo de servicios y productos de bibliotecas: al igual que en las tareas anteriores, el mercadeo es una actividad poderosamente centrada en el usuario, por lo tanto, se le debe estudiar para generar actividades y estrategias de promoción efectivas. El mercadeo parte de la administración estratégica, por lo tanto, a través de design thinking pueden estudiarse tanto sus procesos como sus productos.

- Administración de bibliotecas: para esta tarea, el design thinking puede utilizarse para mejorar e innovar procesos, lo cual impacta a la biblioteca como sistema de información.

- Estudio de usuarios: además de la entrevista a profundidad, el design thinking propone otras técnicas para "empatizar" o conocer a los usuarios, entre ellas el customer jouney y personas. 
- Alfabetización mediática e informacional: para esta labor, se propone el design thinking como una metodología para idear servicios acordes a las necesidades reales de los usuarios.

- Investigación: la escritura académica y la investigación son procesos de creación y que requieren de creatividad. La etapa "idear" requiere de este proceso, pero de igual manera se puede utilizar el design thinking como estrategia didáctica para enseñar a escribir textos académicos.

- Creación de servicios para usuarios con discapacidad: considerando que la biblioteca es un espacio de inclusión social y que se adecúa a las necesidades de sus usuarios, en la actualidad es fundamental ofrecer servicios, recursos y espacios que puedan ser aprovechados por todas las personas. Por lo tanto, a través del design thinking se podrían idear y mejorar servicios especializados dirigidos a personas con discapacidad.

- Desenvolvimiento en makerspaces: el design thinking propone que los individuos diseñen prototipos y utilicen materiales, recursos y artefactos de forma creativa para tales efectos, además esta metodología estimula el "aprender haciendo", por lo tanto, se promueve como una estrategia idónea para que los profesionales en bibliotecología aprovechen los espacios de co-trabajo o los makerspaces y asimismo aprendan a utilizar los recursos que allí pueden disponer.

Finalmente, es destacable que la metodología fomenta el desarrollo de habilidades blandas tales como la comunicación, el trabajo en equipo y el respeto a opiniones ajenas, habilidades muy requeridas por la realidad laboral actual. Es importante mencionar además que la mayoría de las iniciativas de su aplicación en bibliotecología son provenientes de Estados Unidos, un país que a través de los años se ha caracterizado por su constante innovación en la disciplina.

\section{Referencias}

Armstrong, C. (2016). Teaching innovation through empathy: Design thinking in the undergraduate business classroom. Management Teaching Review, 1 (3). DOI https://doi.org/10.1177/2379298116636641 
Beckman, S. y Barry, M. (2007). Innovation as a learning process: Embedding design thinking. California Management Review, 50(1). DOI https://doi.org/10.2307/41166415

Bowler, L. (2014). Creativity through "maker": Experiences and design thinking in the education of librarians. Knowledge Quest, 42 (5).

Buchanan, R. (1992). Wicked problems in design thinking. Design Issues, 18 (2). Recuperado de http://web.mit.edu/jrankin/www/engin as lib art/Design thinking.pdf

Clarke, R., Bell, S. (2018). Transitioning from the MLS to the MLD: Integrating design thinking and philosophy into library and information science education. En Re-envisioning the MLS: Perspectives on the future of Library and Information Science Education (Advances in Librarianship, Volume 44A), editado por Johnna Percell, Linday C. Sarin, Paul T. Jaeger y John Carlo Bertot. Estados Unidos: Emerald Publishing. Recuperado de https://surface.syr.edu/cgi/viewcontent.cgi?article=1174 HYPERLINK "https://surface.syr.edu/cgi/viewcontent.cgi?article=1174\&context=istpub"\& HYPERLINK

"https://surface.syr.edu/cgi/viewcontent.cgi?article=1174\&context=istpub"context=istpub

Coleman, M.C. (2016). Design thinking and the school library. Knowledge Quest, 44 (5). Recuperado de https://files.eric.ed.gov/fulltext/EJ1099478.pdf

Escobar, M., Infante, M. (2018). La formación competente del profesional de la bibliotecología y las ciencias de la información. Transformación 14 (1). Recuperado de http://scielo.sld.cu/scielo.php?script=sci abstract HYPERLINK "http://scielo.sld.cu/scielo.php?script=sci abstract\&pid=S2077-29552018000100002"\& HYPERLINK "http://scielo.sld.cu/scielo.php?script=sci abstract\&pid=S2077$29552018000100002 "$ pid=S2077-29552018000100002

Gardner, L. (2017a). How design thinking can be applied across the campus. The Chronicle of Higher Education. Recuperado de https://www.chronicle.com/article/How-DesignThinking-Can-Be/241127

Gardner, L. (2017b). Can design thinking redesign higher ed? The Chronicle of Higher Education. Recuperado de_https://www.chronicle.com/article/Can-Design-ThinkingRedesign/241126

Hasso Plattner Institute of Design at Stanford (s.f). Miniguía: Una introducción al design thinking. Traducido por Felipe González. Recuperado de https://dschool- 
old.stanford.edu/sandbox/groups/designresources/wiki/31fbd/attachments/027aa/GU\% C3\%8DA\%20DEL\%20PROCESO\%20CREATIVO.pdf?sessionID=8af88fee76ecd1fb78 79c915073461486c425622

IDEO (2013). Design thinking for educators. Recuperado de https://designthinkingforeducators.com/

IDEO (2014). Design thinking for libraries: A toolkit for patron-centered design. Recuperado de http://designthinkingforlibraries.com/

Interaction Design Foundation (2018a). Design thinking: Get a quick overview of the history.

Recuperado de https://www.interaction-design.org/literature/article/design-thinking-geta-quick-overview-of-the-history

Interaction Design Foundation (2018b). Design thinking: A quick overview. Recuperado de https://www.interaction-design.org/literature/article/design-thinking-a-quick-overview

Koh, J., Chai, C., Wong, B., Hong, H. (2015). Design thinking for education: Conceptions and applications in teaching and learning. Singapore: Springer.

Leverenz, C. (2014). Design thinking and the wicked problem of teaching writing. Computers and Composition, 33. DOI https://doi.org/10.1016/j.compcom.2014.07.001

Lor, R. (2017). Design thinking in education: A critical review of literature. En: Asian Conference on Education \& Psychology, Bangkok, Thailandia. Recuperado de https://www.researchgate.net/publication/324684320 Design Thinking in Education A Critical Review of Literature

Luka, I. (2014). Design thinking in pedagogy. Journal of Education Culture and Society, 2. Doi:10.15503/jecs20142.63.74

Monfasani, R. M. (2016). Introducción a la bibliotecología. Argentina: Alfagrama.

Mora, M. (2010). La teoría de la mente en el contexto de la teoría dinámica de la tropa y sus aportes a la educación. Revista Educación, 32 (2). Recuperado de https://revistas.ucr.ac.cr/index.php/educacion/article/view/489/483

Newberry, J. (2016). Adventures in teaching millennials: Population health, community engagement, and design thinking for first year medical students. Annals of Emergency Medicine, 68 (4).

Organización de las Naciones Unidas para la Educación, la Ciencia y la Cultura (2009). Conferencia Mundial sobre la Educación Superior - 2009: La nueva dinámica de la 
educación superior y la investigación para el cambio social y el desarrollo. París. Recuperado de http://www.unesco.org/education/WCHE2009/comunicado es.pdf

Pirela, J., Pulido, N. (2017). Tendencias para la educación superior en sistemas de información, bibliotecología y archivística. Opción: Revista de Ciencias Humanas y Sociales, 33 (82), Recuperado de https://dialnet.unirioja.es/servlet/articulo?codigo=6233633

Ríos-Ortega, J. (2008). Didáctica de la bibliotecología: Teoría y principios desde la enseñanza de la ciencia. México: Universidad Autónoma de México.

Rosa, K., Storey, T. (2016). American libraries in 2016: creating their future by connecting, collaborating and building community. IFLA Journal, 42 (2).

Sabor, J. (1968). Métodos de enseñanza de la bibliotecología. Ginebra, Suiza: UNESCO.

Stickdorn, M., Schneider, J. (2011). This is service design thinking. New Jersey, Estados Unidos: Wiley.

Van de Grift, T., Kroeze, R. (2016). Design thinking as a tool for interdisciplinary education in health care. Academic Medicine 21(9).

Vergara, G., Cuentas, H. (2015). Actual vigencia de los modelos pedagógicos en el contexto educativo. Opción, 31(6). Recuperado de http://www.redalyc.org/pdf/310/31045571052.pdf

\section{Notas sobre la autora}

Daniela Rodríguez Valerio. Licenciada en Bibliotecología y Ciencias de la Información por la Universidad de Costa Rica. Además, es Bachiller en Bibliotecología con énfasis en Bibliotecas Educativas. En la actualidad, es académica de la Escuela de Bibliotecología y Ciencias de la Información, Universidad de Costa Rica. Correo electrónico daniela.rodriguezvalerio@gmail.com, ORCID ID: https://orcid.org/0000-0003-3737-8851 